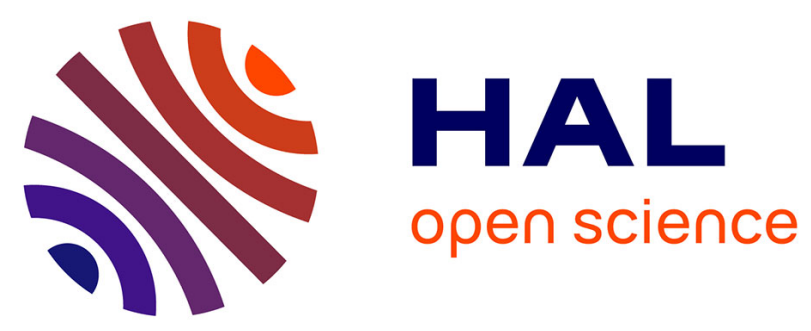

\title{
Investigating the low-frequency vibrations of chlorophyll derivatives using terahertz spectroscopy
}

Dominique Coquillat, Emma O'Connor, Etienne Brouillet, Yoann Meriguet, Cédric Bray, David J. Nelson, Karen Faulds, Jeremie Torres, Nina Diakonova

\section{- To cite this version:}

Dominique Coquillat, Emma O'Connor, Etienne Brouillet, Yoann Meriguet, Cédric Bray, et al.. Investigating the low-frequency vibrations of chlorophyll derivatives using terahertz spectroscopy. SPIE Optical Engineering + Applications, 2021, San Diego, California, United States, Aug 2021, San Diego, United States. pp.118270F, 10.1117/12.2593589 . hal-03406165

\section{HAL Id: hal-03406165 https://hal.science/hal-03406165}

Submitted on 23 Nov 2021

HAL is a multi-disciplinary open access archive for the deposit and dissemination of scientific research documents, whether they are published or not. The documents may come from teaching and research institutions in France or abroad, or from public or private research centers.
L'archive ouverte pluridisciplinaire HAL, est destinée au dépôt et à la diffusion de documents scientifiques de niveau recherche, publiés ou non, émanant des établissements d'enseignement et de recherche français ou étrangers, des laboratoires publics ou privés. 


\title{
Investigating the low-frequency vibrations of chlorophyll derivatives using terahertz spectroscopy
}

\author{
Dominique Coquillat*a , Emma O'Connor ${ }^{\mathrm{b}}$, Etienne V. Brouillet ${ }^{\mathrm{c}}$, Yoann Meriguet ${ }^{\mathrm{a}, \mathrm{d}}$, Cedric Bray ${ }^{\mathrm{a}}$, \\ David J. Nelson ${ }^{\mathrm{c}}$, Karen Faulds ${ }^{\mathrm{b}}$, Jeremie Torres ${ }^{\mathrm{d}}$, Nina Dyakonova ${ }^{\mathrm{a}}$ \\ ${ }^{a}$ Laboratoire Charles Coulomb (L2C), Université de Montpellier, CNRS, Montpellier, France ; \\ ${ }^{\mathrm{b}}$ Centre for Molecular Nanometrology, WestCHEM, Department of Pure and Applied Chemistry, \\ Technology and Innovation Centre, University of Strathclyde, UK; \\ ${ }^{c}$ WestCHEM Department of Pure and Applied Chemistry, University of Strathclyde, Glasgow, UK; \\ ${ }^{\mathrm{d}}$ Institut d'Electronique et des Systèmes (IES), Université de Montpellier, CNRS, France
}

\begin{abstract}
The terahertz absorption spectra of sodium magnesium chlorophyllin (Chl-Mg-Na) and sodium copper chlorophyllin $(\mathrm{Cu}-\mathrm{Chl})$, two major members of the chlorophyll derivative family, have been measured in the range $0.2-3.0 \mathrm{THz}$ $\left(6.6-100 \mathrm{~cm}^{-1}\right)$, at room temperature. Additionally, surface-enhanced Raman scattering spectroscopy was used to supplement data in the higher frequency range. The capability of terahertz spectroscopy for quantitative characterization of Chl-Mg-Na intermolecular vibrations was investigated and the sensitivity of the $1.82-\mathrm{THz}$ feature with degree of hydration by changes in the molecular environment was examined. For $\mathrm{Cu}-\mathrm{Chl}$ derivative, a broad feature was observed around $1.8 \mathrm{THz}$ which currently hinders clear $\mathrm{Cu}-\mathrm{Chl}$ identification and quantification.
\end{abstract}

Keywords: chlorophyll, sodium magnesium chlorophyllin, sodium copper chlorophyllin, terahertz time-domain spectroscopy, intermolecular vibrations, surface-enhanced Raman spectroscopy, mass spectroscopy

\section{INTRODUCTION}

Terahertz (THz) spectroscopic techniques have proven to be more sensitive than other techniques by revealing unique $\mathrm{THz}$ spectra in the $0.1-3.5 \mathrm{THz}$ frequency range, arising from the molecular nature of their chemical and crystal structures. Consequently, many molecular crystals have been investigated using $\mathrm{THz}$ techniques. ${ }^{1-3}$ Semisynthetic chlorophyll derivatives have many advantages over natural chlorophylls, such as good photo and thermal stabilities and excellent photosensibility. Hence chlorophyll derivative based materials and devices are already prospected. For instance, spontaneous supramolecular assembly systems were accessed and show promising results for applications such as artificial photosynthesis. ${ }^{4}$ Recently, a new type of biosolar cells using semisynthetic chlorophyll derivatives as photoactive layers to improve the conversion from light energy to electricity was developed ${ }^{5}$, and chlorophyll-based transistors for potential bioelectronics applications were demonstrated. ${ }^{6}$ Sodium magnesium chlorophyllin (Chl-Mg-Na) and sodium copper chlorophyllin $(\mathrm{Cu}-\mathrm{Chl})$ are two major members of the semisynthetic derivatives of the natural $\mathrm{Chl}-a$. Their molecular structures are shown in the insets of Figs. 1 and 4, respectively. Most previous reports on natural chlorophylls and on semisynthetic derivatives are focusing the ultraviolet-visible and infrared regions of the electromagnetic spectrum. Few studies have investigated the polycrystalline materials in the lower frequency region $(0.3-3.0 \mathrm{THz})$, where intermolecular and intramolecular vibrations are both present, creating complex vibrational modes. Thus, the low-frequency vibrations could serve as spectral "fingerprints" and be used for identification, detection, characterisation and quantification of chlorophyll derivatives. In this study, we used time-domain spectroscopy (THzTDS) to cover the spectral absorption range from 0.2 to 2.5 in order to present low-frequency vibrations of two major chlorophyll derivatives. Additionally, surface-enhanced Raman scattering (SERS) spectra of chlorophyllin in the presence of aggregated silver nanoparticles (AgNPs) were measured to analyze intramolecular vibrations at higher frequencies. The quantitative capability of $\mathrm{THz}$ spectroscopy was investigated. Additionally, the changes in molecular environment caused by differences in hydration levels was detected, demonstrating the sensitivity of THz spectroscopy.

*dominique.coquillat@umontp.fr; phone +33467149415 


\section{METHODS}

Powdered sodium magnesium chlorophyllin and sodium copper chlorophyllin (commercial grade) were purchased from Catalyons and Alfa Aesar, respectively and used without further purification. The samples used for SERS spectroscopy were dispersed in water or methanol at appropriate mass ratios. For SERS measurements, a dispersion of AgNPs in water was added to the chlorophylline solutions. For THz-TDS measurements, the Chl-Mg-Na samples used were prepared by mixing as-received powder with high-density polyethylene (HDPE) and pressing the mixture into approximately $1 \mathrm{~mm}$ thick pellets with a $13 \mathrm{~mm}$ diameter. Pure Chl-Mg-Na powder were also compressed into thinner pellets. The $\mathrm{Cu}-\mathrm{Ch}$ powder was recrystallised in water. The newly recrystallised $\mathrm{Cu}-\mathrm{Chl}$ crystals were mixed with $\mathrm{HDPE}$ in a 1:1 weight ratio, thinly grinded to form an homogeneous mixture and pelletized.

The spectral coverage of the SERS measurements was from 200-1800 $\mathrm{cm}^{-1}$ (6.0-54 THz) with a $532 \mathrm{~nm}$ excitation light. This excitation wavelength falls at higher energy than the $\mathrm{Q}_{\mathrm{y}}$ absorption band of Chl-Mg-Na (the $\mathrm{Q}_{\mathrm{y}}$ absorption feature was observed at $653 \mathrm{~nm}$ ). The maximum laser power was $40 \mathrm{~mW}$ and the average scan time $1 \mathrm{~s}$. The THz-TDS spectra were acquired using a commercial TeraPulse 4000 instrument across the spectral range of 0.1-3.5 THz (TeraView Ltd, Cambridge, UK) with frequency resolution of $0.002 \mathrm{THz}$. The sample compartment was evacuated to reduce the effect of the water vapor in the spectra, affecting the degree of hydration of the sample. The samples were measured in transmission geometry and the THz-TDS spectra were normalized and had an empirical baseline function subtracted.

\section{RESULTS AND DISCUSSION}

SERS combines the specificity of vibrational Raman spectroscopy with the increased sensitivity provided by plasmon assisted scattering, induced in our case by AgNPs. Figure 1 presents the SERS spectra of Chl-Mg-Na in presence of AgNPs. The absorptions in this frequency range $\left(200-1800 \mathrm{~cm}^{-1}\right)$ are governed by the intramolecular vibrations of the sample. Below $400 \mathrm{~cm}^{-1}$, Chl-Mg-Na exhibits features near 217, 247, 308, 344, and 370-385 $\mathrm{cm}^{-1}$. The comparison with both SERS ${ }^{7}$ and resonant Raman $^{8}$ spectra of Chl- $a$ reveals that the SERS spectrum of Chl-Mg-Na is almost similar. Most of the frequency difference are less than $10 \mathrm{~cm}^{-1}$. In the intramolecular frequency range, the SERS spectra of the natural Chl- $a$ and of Ch-Mg-Na being qualitatively similar suggests that the presence of the features at 308 and $344 \mathrm{~cm}^{-1}$ in the Chl-Mg-Na spectrum can be assigned to $\mathrm{MgN}_{4}$ vibrations.

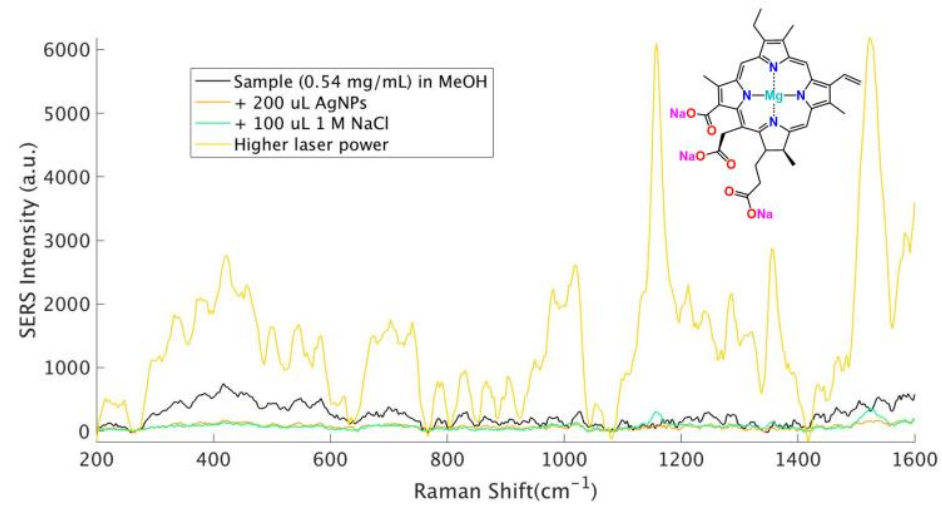

Figure 1. SERS spectrum of Chl-Mg-Na in presence of AgNPs in a water:MeOH mixture at room temperature. Inset: Molecular structure of sodium magnesium chlorophyllin (Chl-Mg-Na)

Below $3.0 \mathrm{THz}\left(100 \mathrm{~cm}^{-1}\right)$, the THz-TDS technique is more effective for the observation of the mixed intermolecular and intramolecular frequencies modes. The THz-TDS absorption spectra were obtained for different concentrations of ChlMg-Na powder in HDPE and reported in Fig. 2 a). The spectra are normalized and have an empirical baseline function subtracted. The position, amplitude and full-width of the absorption peaks were determined by a multipeak-fit using Lorentzian function. Four clear resonances are observed at $1.44 \mathrm{THz}\left(48.1 \mathrm{~cm}^{-1}\right), 1.64 \mathrm{THz}\left(54.6 \mathrm{~cm}^{-1}\right), 1.82 \mathrm{THz}(61.3$ $\left.\mathrm{cm}^{-1}\right)$, and $2.03 \mathrm{THz}\left(67.7 \mathrm{~cm}^{-1}\right)$. The relatively strong and well-resolved features present an opportunity for quantitative analysis. Therefore, we investigated the concentration dependence of the three stronger features. Figure 2 b) presents the variation of absorption as a function of the amount of Chl-Mg-Na contained in the 13-mm diameter pellets with a linear fit crossing zero. As expected, the absorption increases linearly with Chl-Mg-Na amount and appears to obey the BeerLambert law for all three features. 

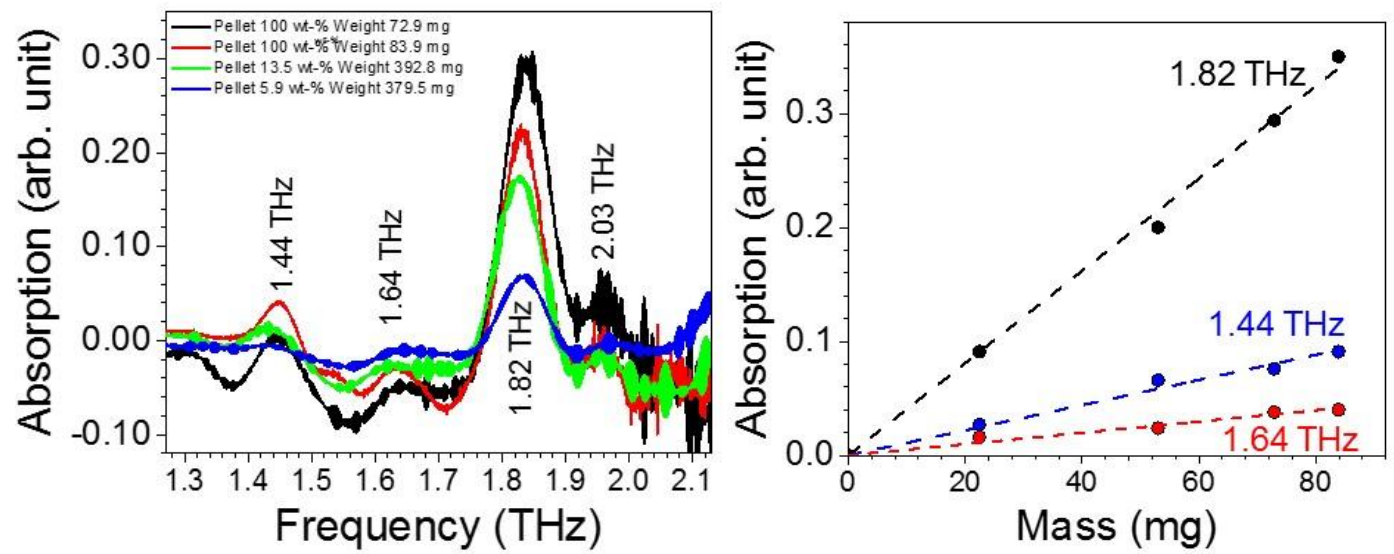

Figure 2. a) THz spectra of pure Chl-Mg-Na pellets (72.6 mg and $83.9 \mathrm{mg}$ ) and $5.9 \mathrm{wt}-\%$ and $13.5 \mathrm{wt}-\% \mathrm{Chl}-\mathrm{Mg}-\mathrm{Na}$ in HDPE pellets (with total weight $379.5 \mathrm{mg}$ and $392.8 \mathrm{mg}$, respectively). The spectra were baseline corrected. b) Absorption as a function of Chl-Mg-Na weight in the 13-mm diameter pellet of selected features at $1.44 \mathrm{THz}, 1.64 \mathrm{THz}$, and $1.82 \mathrm{THz}$.

To better understand intermolecular interactions and crystalline structures, a method using small amounts of solvents to significantly change the molecular environment, hence affect the intermolecular vibrations was previously demonstrated. ${ }^{9}$ Here, we have observed the position change of the THz feature at $1.82 \mathrm{THz}$ by changing the degree of hydration. Water in the Chl-Mg-Na polycrystal was easily removed under vacuum. Fig. 3 a) shows the time-dependent $\mathrm{THz}$ feature during dehydration. As the sample dries, the 1.82- $\mathrm{THz}$ feature decreases in intensity and the position shifts towards lower frequencies. It should be noted that no drastic change was observed for the $1.44-\mathrm{THz}$ feature. The peak position of the 1.82-THz-feature is reported on Fig. 3 b). After 8 hours of vacuum dehydration, the feature position remains nearly unchanged.
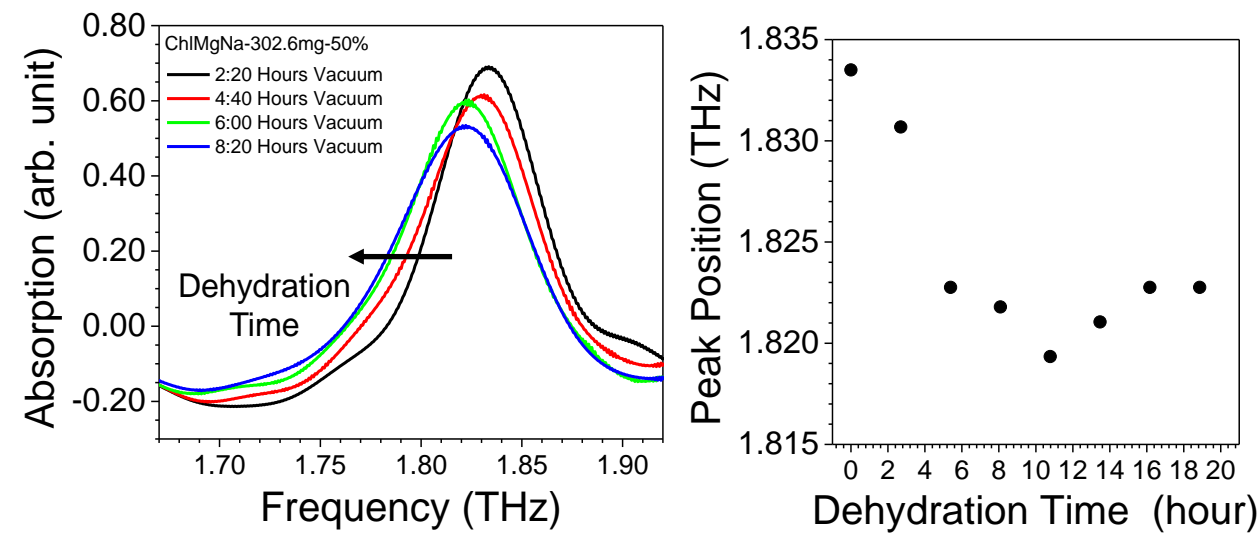

Figure 3. a) The time-dependent change of the $1.82-\mathrm{THz}$ feature as a function of dehydration time under vacuum of a pellet containing $50 \mathrm{wt}-\%$ Chl-Mg-Na in dehydrated HDPE (total weight $302.6 \mathrm{mg}$ ). The spectra were baseline corrected. b) Feature position as a function of dehydration time.

For comparison studies with Chl-Mg-Na, we investigated the low-frequency vibrations of the $\mathrm{Cu}-\mathrm{Chl}$ derivative obtained from two different suppliers. In each case, when the powder was measured in pellet form and without any further recrystallization, we have obtained featureless spectra. However, after a first attempt of recrystallization using water as solvent, a broad feature was observed around $1.8 \mathrm{THz}$. Currently, this broad feature behavior hinders clear $\mathrm{Cu}-\mathrm{Chl}$ identification and quantification. Several studies reported that commercially grade preparations of $\mathrm{Cu}$-Chl were in fact a complex mixture of different chlorin molecules. ${ }^{10}$ Therefore, in addition to the more common copper derivative shown in the inset of Fig. 4, other molecules may be present. This also includes the presence of different metals or metal-free derivatives. The complex mixture of different molecules may explain why well-resolved vibration modes were not observed for the recrystallized $\mathrm{Cu}-\mathrm{Chl}$ powder. Separation and identification of the components of $\mathrm{Cu}$-Chl powders have to be performed before new $\mathrm{THz}$ characterizations. 


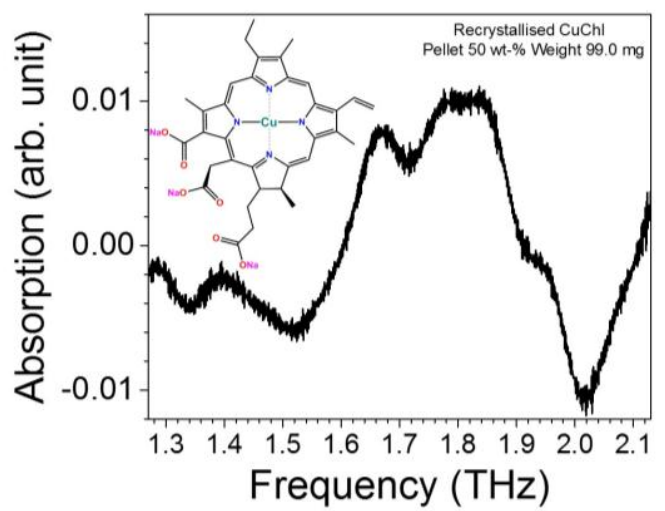

Figure 4. THz spectrum of a pellet containing $50 \mathrm{wt}-\%$ of recrystallized $\mathrm{Cu}-\mathrm{Chl}$ in HDPE (total weight $99.0 \mathrm{mg}$ ). Inset: Molecular structure of copper chlorophyllin (Cu-Chl).

\section{CONCLUSIONS}

$\mathrm{THz}$ spectroscopy appears to be a really good candidate to probe the frequencies of the intermolecular vibrations for chlorophyll derivatives, their shifts and amplitude variations with the degree of hydration. The interpretation of the results is phenomenological, meaning that detailed assignments of individual features in the $\mathrm{THz}$ spectra need to be established using modelling support. The measured $\mathrm{THz}$ spectra of these chlorophyll derivatives are a significant contribution to the collection of biomolecular crystal spectra being currently accumulated in the literature. Extension of such investigations to chlorophyll in plants offers a rich but challenging field of study. ${ }^{11}$

Acknowlegments: This project is supported by the Contrat de Plan Etat-Région (CPER)-Région Occitanie "PlantEnvi", by the Montpellier University of Excellence I-Site MUSE (PRIME@MUSE), and by CNRS through International Research Project "TeraMIR". The authors thank Catalyons Laboratories for providing chlorophyll derivative powders

\section{REFERENCES}

[1] Jepsen, P.U., Cooke, D.G., Koch, M., "Terahertz spectroscopy and imaging, modern techniques and applications," Laser Photon. Rev. 5 (2011).

[2] Bawuah, P., Zeitler, J. A., "Advances in terahertz time-domain spectroscopy of pharmaceutical solids: A review," Trends in Analytical Chemistry 139116272 (2021).

[3] Ruggiero, M., Zeitler, J. A., "Resolving the origins of crystalline anharmonicity using terahertz Time-Domain Spectroscopy and ab Initio Simulations,” J. Phys. Chem. B, 120, 11733 (2016).

[4] Matsubara, S., Tamiaki, H., "Photoactivated supramolecular assembly using caged chlorophylls for the generation of nanotubular self-aggregates having controllable lengths," ACS Appl. Nano Mater. 3, 1841 (2020).

[5] Duan,S., Zhou, Q., Li, A., Wang, X-F., Sasaki,S.I. and Tamiaki, H., "Semisynthetic chlorophyll derivatives toward solar energy applications," Sol. RRL 4, 2000162 (2020)

[6] Yu, Y., Zhang, Y., Jin, L., Chen, Z., Li, Y., Li, Q., Cao, M., Che, Y., Dai, H., Yang, J., Yao, J., "Photoelectricity and thermoelectricity in organic chlorophyll phototransistors," Organic Electr, 65, 381 (2019).

[7] Thomas, L., Kim, J-H., Cotton, T., "Comparative study of resonance Raman and surface-enhanced resonance Raman Chlorophyll a spectra using Soret and red Excitation,” J. Am. Chem. Soc. 112, 9379 (1990).

[8] Zhou, C., Diers, J., Bocian, D., "Qy-Excitation resonance Raman spectra of Chl- $a$ and related complexes. Normal mode characteristics of the low-frequency vibrations," J. Phys. Chem. B, 101, 9635 (1997).

[9] Nagai, N., Kumazawa, R., Fukasawa, R., "Direct evidence of inter-molecular vibrations by THz spectroscopy." Chemical Physics Letters 413: 495 (2005).

[10] Tumolo, T., Lanfer-Marquez, U. M., “Copper chlorophyllin: A food colorant with bioactive properties?,” Food Research International 46, 451 (2012).

[11] Abautret, Y., Coquillat, D., Zerrad, M., Buet, X., Bendoula, R., Soriano, G., Brouilly, N., Héran, D., GrèzesBesset, et al.., "THz probing of sunflower leaf multilayer organization," Opt Express 28, 35018 (2020). 